05

\title{
Магнитные взаимодействия в структуре фазы Ауривиллиуса $\mathrm{Bi}_{5} \mathrm{FeTi}_{3} \mathrm{O}_{15}$
}

\author{
() С.Г. Ястребов, Н.А. Ломанова \\ Физико-технический институт им. А.Ф. Иофрфе РАН, \\ Санкт-Петербург, Россия \\ E-mail: yastrebov@mail.ioffe.ru \\ Поступила в Редакцию 27 октября 2021 г. \\ В окончательной редакции 27 октября 2021 г. \\ Принята к публикации 28 октября 2021 г.
}

\begin{abstract}
Анализируются зависимости от температуры FC и ZFC намагниченности мультиферроика $\mathrm{Bi}_{5} \mathrm{FeTi}_{3} \mathrm{O}_{15}$ со структурой четырехслойной фазы Ауривиллиуса. Ход зависимостей и осцилляции их производных по температуре от FC и ZFC могут отражать влияние на магнитные свойства зерен порошка этого материала. Отсутствие выраженного температурного порога на зависимостях $\mathrm{FC}(\mathrm{ZFC})$ можно связать с преимущественным влиянием на характер намагничивания образца спинового кантирования, вызванного взаимодействием Дзялошинского-Мория.
\end{abstract}

Ключевые слова: фазы Ауривиллуса, перовскиты, магнитные свойства.

DOI: 10.21883/FTT.2022.02.51931.228

\section{1. Введение}

Мультиферроики $\mathrm{Bi}_{m+1} \mathrm{Fe}_{m-3} \mathrm{Ti}_{3} \mathrm{O}_{3 m+3}$ со структурой фаз Ауривиллиуса являются перспективными для устройств магнитной памяти (FeRAM), как материалы для спинтроники и фотовольтаики [1-4].

В общем случае, кристаллическая структура фаз Ауривиллиуса представляет собой чередование висмуткислородных слоев $F=\left\{\left(\mathrm{Bi}_{2} \mathrm{O}_{2}\right)^{2+}\right\}_{\infty}$ с перовскитоподобными блоками $P=\left\{\left(\mathrm{Bi}_{m+1} \mathrm{Fe}_{m-3} \mathrm{Ti}_{3} \mathrm{O}_{3 m+1}\right)\right\}^{2-}$, состоящими из различного числа слоев $m$ на основе мультиферроика $\mathrm{BiFeO}_{3}$. Соответственно, элементарная ячейка $\mathrm{Bi}_{5} \mathrm{FeTi}_{3} \mathrm{O}_{15}$ содержит $m=4$ слоя в перовскитоподобном блоке (рис. 1).

В работах [3-5] установлено, что распределение ионов $\mathrm{Fe} / \mathrm{Ti}$ в структуре фаз Ауривиллиуса с $m \leq 5$ является упорядоченным и катионы железа заселяют, преимущественно, позиции $B(1)$ во внутренних слоях перовскитоподобного, а катионы титана - позиции $B(2)$ на внешних его слоях (рис. 1). Характер этого распределения влияет на устойчивость материалов на их основе и коррелирует с изменением их функциональных, в том числе магнитных, характеристик [2-4,6-8]. Магнитное поведение фаз Ауривиллиуса на сегодняшний день является предметом дискуссий, поскольку несмотря на накопленный экспериментальный опыт (см., например, обзор [1] и ссылки в нем), нет единой трактовки наблюдаемого магнитного отклика. Поэтому изучение локального взаимодействия магнитных катионов в таких структурах является актуальной задачей.

Экспериментальные результаты, приведенные в работах [4,9-11], выявляют трудности синтеза материалов на основе соединений $\mathrm{Bi}_{m+1} \mathrm{Fe}_{m-3} \mathrm{Ti}_{3} \mathrm{O}_{3 m+3}$ с $m>5$, независимо от выбранной технологии. Формирование в них примесных фаз на основе системы $\mathrm{Bi}_{2} \mathrm{O}_{3}-\mathrm{Fe}_{2} \mathrm{O}_{3}$ [12] затрудняет трактовку магнитного отклика. Поэтому с фундаментальной точки зрения четырехслойное соединение $\mathrm{Bi}_{5} \mathrm{Ti}_{3} \mathrm{FeO}_{15}$ является удобным модельным объектом для уточнения особенностей намагниченности фаз Ауривиллиуса, поскольку в материалах на его основе выход целевого продукта максимален.

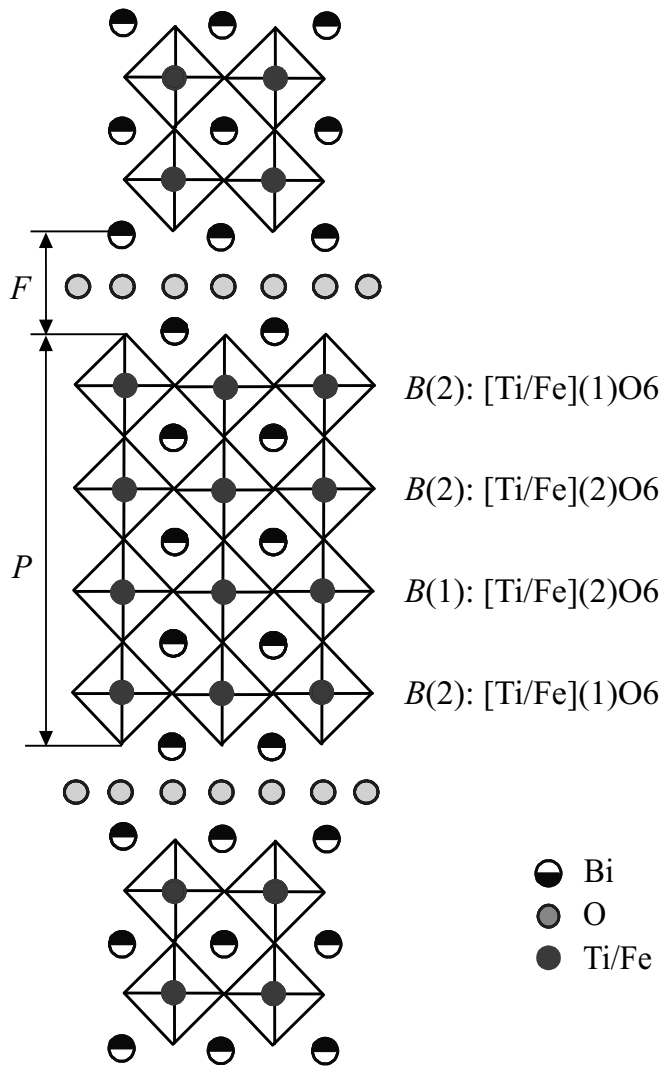

Рис. 1. Схема расположения слоев в структуре $\mathrm{Bi}_{5} \mathrm{FeTi}_{3} \mathrm{O}_{15}$. 
Как показано в $[4,5]$, соединение $\mathrm{Bi}_{5} \mathrm{FeTi}_{3} \mathrm{O}_{15}$ парамагнитно при комнатной температуре и для него характерно ближнее магнитное упорядочение. В работе [7] магнитное поведение $\mathrm{Bi}_{5} \mathrm{FeTi}_{3} \mathrm{O}_{15}$ описывается как близкодействующее (локализованное) антиферромагнитное взаимодействие. Как отмечают многие исследования (см., например, обзор [1] и ссылки в нем), для технических применений перспективны материалы, в которых может быть достигнут магнитный порядок выше комнатной температуры. При этом высказывается предположение, что фаза Ауривиллиуса $\mathrm{Bi}_{5} \mathrm{FeTi}_{3} \mathrm{O}_{15}$ с $m=4$ к таким материалам не относится. Теоретических моделей намагниченности $\mathrm{Bi}_{5} \mathrm{FeTi}_{3} \mathrm{O}_{15}$ сравнительно немного. Основные представлены в работах $[7,8]$ и описывают особенности связей ионов $\mathrm{Fe}^{3+}$ в структуре с соседними атомами, вносящими вклад в магнетизм.

В настоящей работе впервые выполнен анализ экспериментальной зависимости намагниченности $\mathrm{Bi}_{5} \mathrm{Ti}_{3} \mathrm{FeO}_{15}$ от температуры в сравнении с теоретическими данными и обсуждена ее природа.

\section{2. Методика эксперимента}

Материал синтезирован методом твердофазных химических реакций. Исходными реагентами для синтеза были оксиды висмута, железа (III) и титана (IV), взятые в стехиометрии соединения $\mathrm{Bi}_{5} \mathrm{FeTi}_{3} \mathrm{O}_{15}$. Подробно технология описана в [9]. Для измерения намагниченности был использован образец в виде поликристаллического порошка.

Фазовый состав определялся на дифрактометре Shimadzu XRD-7000. Использовалось $\mathrm{CuK}_{\alpha}$ излучение длиной волны $\lambda=1.541 \AA$. Уточнение рентгеновской

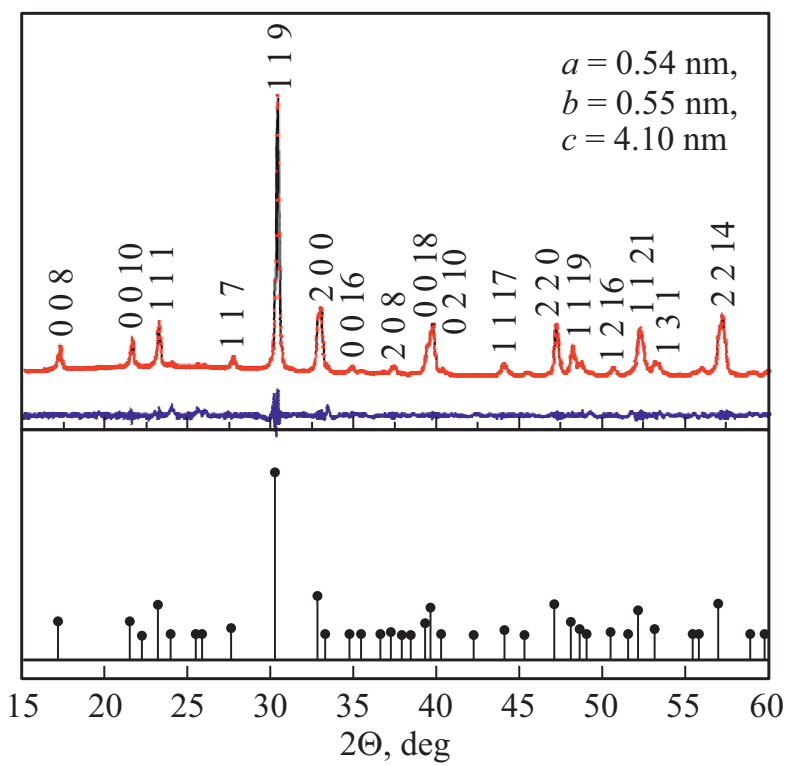

Рис. 2. Рентгеновская дифрактограмма $\mathrm{Bi}_{5} \mathrm{FeTi}_{3} \mathrm{O}_{15}$. Верхняя панель - экспериментальные данные, нижняя - справочные данные по положению пиков (JCPDS Card No. 01-089-8545), пространственная группа А21am. дифрактограммы методом фундаментальных параметров показало хорошее соответствие профиля линии экспериментальным данным. Параметры ромбической элементарной ячейки согласуются с известными данными и приведены на рис. 2.

Элементный состав определялся методом энергодисперсионного микроанализа (сканирующий электронный микроскоп FEI Quanta 200 с приставкой EDAX). Данные рентгенофазового и элементного анализа показали, что полученный материал однофазен и состав целевого продукта соответствует стехиометрическому.

Магнитные измерения проводились на вибрационном магнитометре PPMS Quantum Design в диапазоне температур 5-300 K. Температурные зависимости удельной намагниченности $M$ в режимах zero field cooling (ZFC) и field cooling (FC) определялись при внешнем поле $H=500$ Oe.

\section{3. Экспериментальные результаты}

Результаты магнитного эксперимента полученного материала представлены на рис. 3,4. Для интерпретации магнитных взаимодействий в структуре $\mathrm{Bi}_{5} \mathrm{FeTi}_{3} \mathrm{O}_{15}$ мы используем следующий ход рассуждений. Для количественного описания магнитных характеристик используется модель неупорядоченного квазидвумерного магнетика Гейзенберга при слабом взаимодействия между катионами соседних слоев: в случае $\mathrm{Bi}_{5} \mathrm{FeTi}_{3} \mathrm{O}_{15}$ его элементарная ячейка содержит четыре перовскитоподобных слоя (рис. 1). При этом, реализуется случай разбавленного магнетика поскольку катионы железа, участвующие в антиферромагнитном взаимодействии, замещаются катионами титана, что приводит к их ферромагнетизму.

В самом деле, в случае, если ближайшими соседями ионов железа являются сами ионы железа $\mathrm{Fe}^{3+}$ (с электронной конфигурацией $d 5$ ), взаимодействующие друг с другом через анион кислорода, то в результате проявляется т.н. сверхобменное взаимодействие между ними согласно правилу Гуденаф-Канамори [13,14], что приводит к образованию антиферромагнитной связи. Катионы титана немагнитны и поэтому в случае их соседства с $\mathrm{Fe}^{3+}$ сверхобменное взаимодействие нарушается, а сам катион железа становится ферромагнитным. Поскольку в идеальном случае каждый из четырех слоев в перовскитоподобном блоке может быть описан простой кубической решеткой с четырьмя слоями вдоль оси $c$, каждая позиция магнитного катиона в ней заполняется с вероятностью $x$. В работе [7] предполагается, что ионы $\mathrm{Fe}^{3+}$ заселяют свои позиции случайным образом, тогда как в работах $[4,5]$ определено, что в структуре соединения $\mathrm{Bi}_{5} \mathrm{FeTi}_{3} \mathrm{O}_{15}$ они локализованы упорядоченно и преимущественно во внутренних позициях перовскитоподобного блока (рис. 1)

Условия перколяции на примере кубической ячейки рассмотрены в работах $[7,8]$, в которых показано, что критическая концентрация магнитных катионов для ее реализации должна быть $x_{c} \approx 0.312$. Согласно структурным особенностям $\mathrm{Bi}_{5} \mathrm{FeTi}_{3} \mathrm{O}_{15}$, порог перколяции 

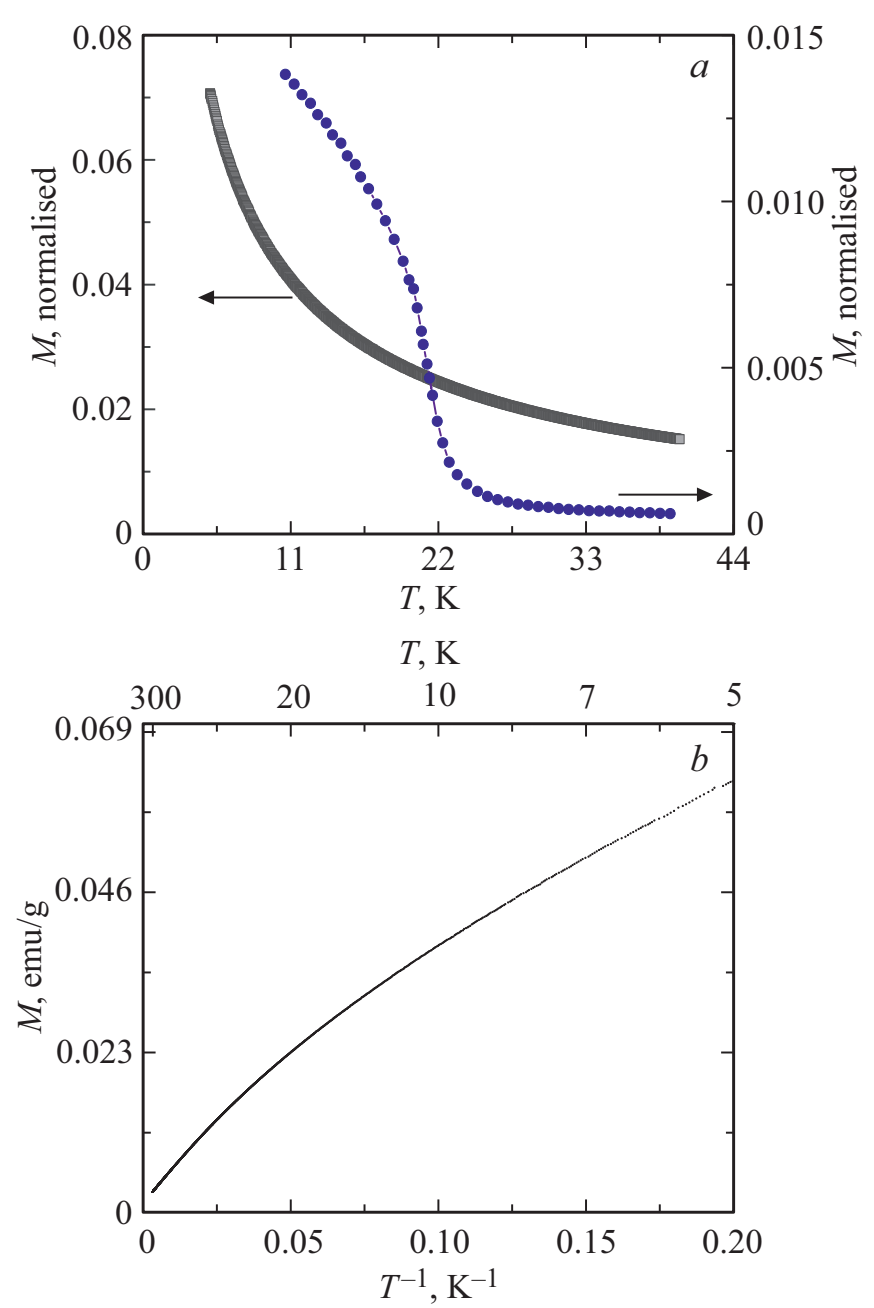

Рис. 3. (a) Зависимости намагниченности FC/ZFC (совпадают) от температуры $(5-300 \mathrm{~K})$, нормированные на площадь под соответствующей кривой, в области $4.2-40 \mathrm{~K}$. Квадратики эксперимент настоящей работы, кружки - расчетные данные работы [7] для системы: $8 n \times n \times n$ элементарных ячеек с $n=4$. (b) Экспериментальные зависимости FC/ZFC (совпадают) от обратной температуры.

для него составляет только $x=0.25$. Поэтому авторы работы [7] исследуют магнетизм локализованных состояний, считая перколяцию невозможной из-за разницы теоретического и экспериментального значения $x$. В рамках сделанных допущений авторы также количественно оценили температурный ход зависимости намагниченности и температуру перехода в немагнитное состояние - приблизительно равна $T \sim 22 \mathrm{~K}$. Зависимость намагниченности от температуры, полученная в нашей работе, как видно из рис. $3, a$, однако, значительно отличается от теоретических предсказаний работы [7]. В частности, на экспериментальной зависимости не заметно резкого падения намагниченности при $T \sim 22 \mathrm{~K}$. Отличия эксперимента и теоретического расчета, скорее всего, проявляются вследствие вклада в ход зависимости других эффектов, отличных от рассмотренных в работе [7]. В самом деле, согласно литературным источникам [15-17], их можно объяснить несколькими механизмами, такими как спиновое кантирование, вызванное взаимодействием Дзялошинского-Мория (ДМ), обменной ферромагнитной связью между ионами $\mathrm{Fe}^{2+}$ и $\mathrm{Fe}^{3+}$ и с обогащенными $\mathrm{Fe}$ нанодоменами, а также не исключается роль обменного взаимодействия между F-центрами [15-17]. Поскольку в рассматриваемом материале $\mathrm{Bi}_{5} \mathrm{FeTi}_{3} \mathrm{O}_{15}$ ионы железа находятся в состоянии $\mathrm{Fe}^{3+}$ [5], наблюдаемые эффекты могут быть связаны, в основном, со спиновым кантированием, вызванным взаимодействием ДМ.

Остановимся на механизмах намагниченности подробнее. Для этого обратимся к рис. 3, $b$, на котором представлена зависимость намагниченности $M$, построенная от обратной температуры для исключения вклада парамагнетизма. Парамагнитный отклик материала, как известно, описывается формализмом Ланжевена и, в случае его проявления, кривая $M(1 / T)$ имела бы вид логистической зависимости. Однако, полученная экспериментальная зависимость таким образом не описывается. Обратим также внимание, что температурные зависимости намагниченности, измеренные как в случае наличия внешнего магнитного поля (FC), так и в его отсутствии (ZFC), совпадают. Это может быть связано с проявлением спонтанной намагниченности, которой не может противодействовать магнитное поле $500 \mathrm{Oe.}$

Из рис. 3 видно, что представленная на нем зависимость - наложение осциллирующей и монотонно падающей с ростом температуры функций, слабый ферромагнетизм сохраняется, вопреки предсказаниям теоретической работы [7], до комнатной температуры. При этом аналогичные зависимости FC и ZFC зависят монотонно от обратной температуры (см. рис. 3).

На рис. 4, $a$ представлена первая производная намагниченности по обратной температуре $\mathrm{m}^{\prime}$. Заметим, что при дифференцировании для сглаживания шума, присущего экспериментальной установке, выполнялось сглаживание экспериментальных точек методом Савитски-Голай [15] с помощью полинома второй степени по окну с десятью опорными точками, при этом степень полинома и количество точек подбирались эмпирически.

Осцилляции можно было бы связать с вкладом в намагниченность магнонов, подвергшихся геометрическому конфайнменту подходящими для этого гранулами порошка рассматриваемого материала, проявляющими ферромагнитные свойства. Такая локализация могла бы привести к осцилляциям на зависимости $m^{\prime}(1 / T)$, что и наблюдается в эксперименте. При этом возбуждение магнонов могло бы происходить по механизму их взаимодействия с фононами решетки материала. Такому взаимодействию посвящена работа [19].

Проверим эту гипотезу. Известно, что дисперсионное уравнение для магнонов имеет вид

$$
\varepsilon=A k^{2},
$$

где $k=2 \pi / \lambda-$ волновой вектор магнона, и $\lambda-$ его длина волны, $A-$ константа. 

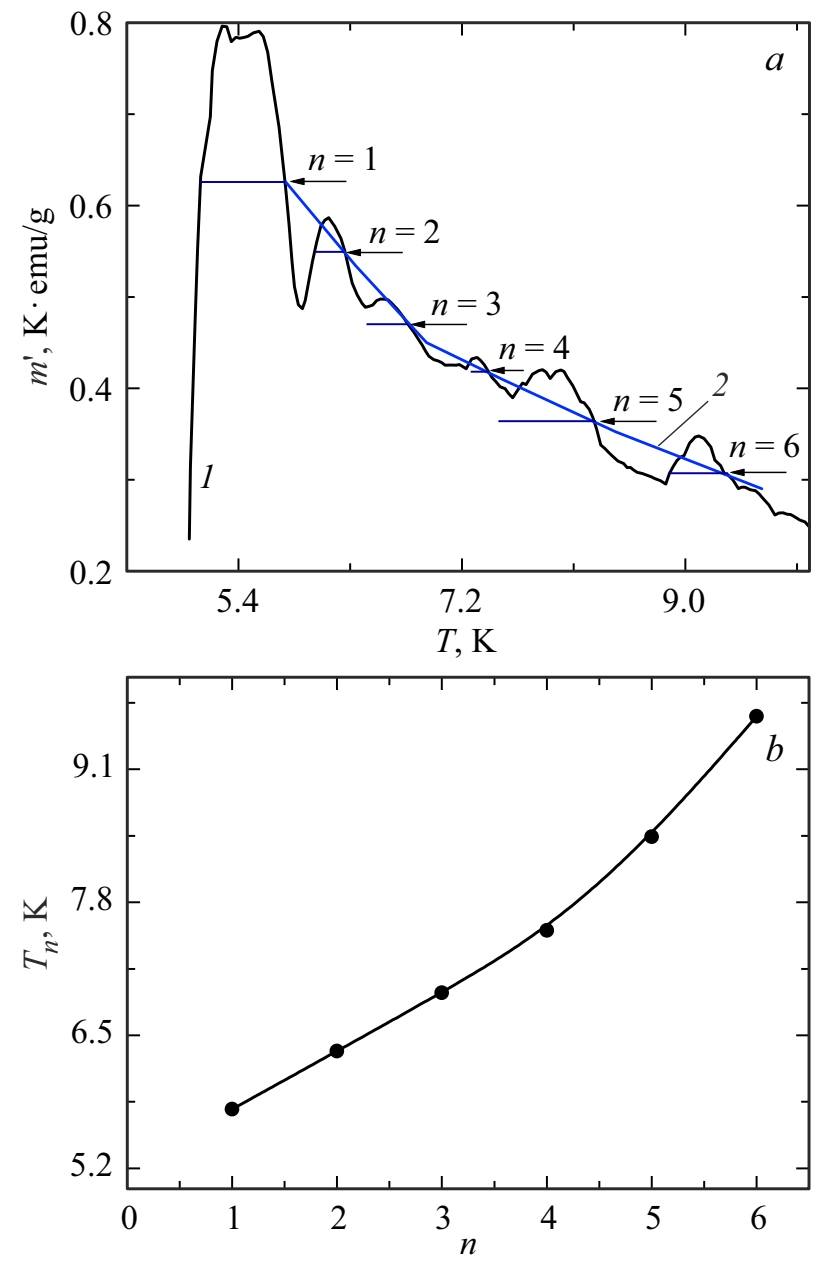

Pис. 4. (a) Зависимость от температуры первой производной $m^{\prime}$ от $M\left(T^{-1}\right)$, представленной на рис. $3, b$, в области проявления особенностей - кривая 1 . Полочки на рисунке среднее геометрическое значение между соседними точками максимумов и минимумов. Кривая $2-$ результат геометрического сглаживания максимальных и минимальных значений осцилляций. Стрелками показаны точки пересечения сглаженной и несглаженной зависимостей. $(b)$ Энергии для точек пересечения рис. $4, a$, отмеченных стрелками, в зависимости от их порядкового номера. Сплошная кривая - параболическая аппроксимация.

В случае простейшей модели локализации магнона на ветке перколяционного кластера длиной $L$ на ней может уложиться полуцелое число длин волн, т.е.

$$
\begin{aligned}
L & =n \frac{\lambda}{2}, \\
k_{n} & =n \frac{\pi}{L}, \\
\varepsilon & =A k_{n}^{2} .
\end{aligned}
$$

Из уравнения (4) следует, что в случае конфайнмента спектр магнонов квантуется. Если предположить, что генерация магнонов связана с температурой решетки и происходит вследствие связи магнонов с фононами, то на зависимости намагниченности от температуры присутствовали бы пики для температур, равных разрешенным энергиям магнона. Заметим также, что при малых значениях $k$ зависимость (4) близка к линейной.

Для проверки гипотезы влияния на намагниченность квантования магнонного спектра рассмотрим следующий подход. Как отмечалось выше, зависимость производной от температуры представляет собой монотонно убывающую с ростом температуры функцию, на которую наложены осцилляции. Т. е. процедура дифференцирования позволила выявить скрытую закономерность зависимости намагниченности от температуры.

Для уточнения значений температуры, при которых на осциллирующем скрытом вкладе в намагниченность наблюдаются экстремумы, на зависимости рис. 4 необходимо выделить монотонно меняющийся с ростом температуры тренд. С этой целью был применен геометрический фильтр, подобный примененному ранее в работе [20] для исследования интерференционной картины в тонких пленках. Его суть состоит в том, что на интервале между последовательно следующими друг за другом минимумами и максимумами осциллирующей интерференционной функции, ее значение заменялось средним геометрическим от максимума и минимума, соответственно. В этом случае отрезок прямой между средними геометрическими значениями, следующими друг за другом, представляет собой нулевой уровень отсчета осцилляций. Результат выполнения этой процедуры для нашего случая показан на рис. 4, $a$. Стрелками и полочками отмечены значения средних геометрических, для удобства через отмеченные значения с помощью аппроксимации полиномом через них проведена линия искомого тренда.

Общеизвестно, что если при переходе через точку экстремума первая производная меняет знак с плюса на минус, то этот экстремум является максимумом осциллирующей функции намагниченности. Заметим, что из рис. 4, $a$ видно, что критерию смены знака при переходе от соседних точек максимума к точке минимума, взяв за начало отсчета кривую тренда, соответствуют все выделенные стрелочками точки. Проведенная через эти точки кривая, как показано на рис. 4, $a$, выявляет общий монотонный тренд $m^{\prime}$ поведения неосциллирующего вклада в намагниченность.

На следующем этапе в зависимости от целого числа $n$ (с увеличением $n$ возрастает энергия), построена зависимость, представленная на рис. 4, $b$. Эта зависимость, в случае конфайнмента магнонов, являлась бы аналогом дисперсии магнонов. Однако такой дисперсионной зависимости нулевому значению волнового вектора соответствовала бы нулевая энергия, в соответствии с выражением (4). Поскольку нулевое значение энергии не достигается на рис. $4, b$, то можно было бы сделать вывод о том, что осцилляции рис. 4, $a$ не связаны с конфайнментом магнонов. Однако, как оказалось, сравнительно недавно похожую зависимость наблюдали в работе [21]. 
Заметим, что к осцилляциям намагниченности может привести взаимодействие ферромагнитных зерен исследуемого порошка $\mathrm{Bi}_{5} \mathrm{FeTi}_{3} \mathrm{O}_{15}$, поскольку порошок исследуемого материала содержит зерна, взаимодействующие между собой. Это взаимодействие подавляется температурой. В первую очередь подавляются наиболее слабо связанные зерна. Подобной моделью в работе [22] объясняются осцилляции намагниченности ферромагнитного графита.

\section{4. Заключение}

В работе рассмотрены особенности магнитных взаимодействий в структуре поликристаллического материала на основе четырехслойного перовскитоподобного соединения $\mathrm{Bi}_{5} \mathrm{FeTi}_{3} \mathrm{O}_{15}$ типа фазы Ауривиллиуса. Показано, что ход зависимостей намагниченности для двух режимов измерения, FC и ZFC, значительно отличается от теоретических предсказаний. В отличие от теории, на экспериментальных данных в диапазоне температур $5-40 \mathrm{~K}$ отсутствует резкий температурный переход в немагнитное состояние при $22 \mathrm{~K}$. Поэтому при конструировании материалов на основе фаз Ауривиллиуса, в которых может быть достигнут магнитный порядок выше комнатной температуры, необходимо учитывать роль различных эффектов, связанных, в первую очередь, со спиновым кантированием, вызванным взаимодействием Дзялошинского-Мория.

На производных экспериментальных FC и ZFC зависимостях намагниченности этого материала от температуры проявляется ряд особенностей, которые могут характеризовать влияние на них специфики намагниченности зерен поликристаллического материала на основе $\mathrm{Bi}_{5} \mathrm{FeTi}_{3} \mathrm{O}_{15}$.

\section{Благодарности}

Авторы благодарят М.П. Волкова (ФТИ им. А.Ф. Иоффе) за магнитометрию полученного материала.

\section{Конфликт интересов}

Авторы заявляют об отсутствии конфликта интересов.

\section{Список литературы}

[1] S. Sun, X. Yin. Crystals 11, 1, 23 (2021).

[2] Y. Huang, G. Wang, S. Sun, J. Wang, R. Peng, Y. Lin, X. Zhai, Z. Fu, Y. Lu. Sci. Rep. 5, 15261 (2015).

[3] T. Pikula, J. Dzik, P. Guzdek, V.I. Mitsiuk, Z. Surowiec, R. Panek, E. Jartych. Ceram. Int. 43, 14, 11442 (2017).

[4] E. Jartych, T. Pikula, M. Mazurek, A. Lisinska Czekaj, D. Czekaj, K. Gaska, J. Przewoznik, C. Kapusta, Z. Surowiec. J. Magn. Magn. Mater. 342, 27 (2013).

[5] N.A. Lomanova, V.G. Semenov, V.V. Panchuk, V.V. Gusarov. J. Alloys Compd. 528, 103 (2012).

[6] N.A. Lomanova, I.V. Pleshakov, M.P. Volkov, V.V. Gusarov. Mater. Sci. Eng. B. 214, 51 (2016).
[7] A.Ya. Birenbaum, A. Scaramucci, C. Ederer. Phys. Rev. B. 95, 104419 (2017).

[8] A.Y. Birenbaum, C. Ederer. Phys. Rev. B. 90, 214109 (2014).

[9] Н.А. Ломанова, М.И. Морозов, В.Л. Уголков, В.В. Гусаров. Неорганан.материалы 42, 2, 189 (2006). [N.A. Lomanova, M.I. Morozov, V.L. Ugolkov, V.V. Gusarov. Inorgan. Mater 42, 2, 189(2006)].

[10] Н.А. Ломанова, М.В. Томкович, А.В. Осипов, В.Л. Уголков, В.В. Панчук, В.Г. Семенов, В.В. Гусаров. Журн. неорган. химии 66, 5, 658(2021). [N.A. Lomanova, M.V. Tomkovich, A.V. Osipov, V.L. Ugolkov, V.V. Panchuk, V.G. Semenov, V.V. Gusarov. Russ. J. Inorgan. Chem., 66, 5, 755 (2021)].

[11] X. Li, Z. Ju, F. Li, Y. Huang, Y. Xie, Z. Fu, R.J. Knize, Y. Lu. J. Mater. Chem. A. 2, 13366 (2014).

[12] O.V. Proskurina, R.S. Abiev, D.P. Danilovich, V.V. Panchuk, V.G. Semenov, V.N. Nevedomsky, V.V. Gusarov. Chem. Eng. Proc. - Proc. Intensif. 143, 107598 (2019).

[13] J.B. Goodenough. Magnetism and the Chemical Bond. Interscience. N. Y. (1963).

[14] J. Kanamori. J. Phys. Chem. Solids 10, 2-3, 87 (1959). doi.org/10.1016/0022-3697(59)90061-7 P

[15] H.Y. Zhao, H. Kimura, Z.X. Cheng, M. Osada, J.L. Wang, X.L. Wang, S.X. Dou, Y. Liu, J.D. Yu, T. Matsumoto, T. Tohei, N. Shibata, Y. Ikuhara. Sci. Rep. 4, 5255 (2014).

[16] H. Sun, X.M. Lu, J. Su, T.T. Xu, C.C. Ju, F.Z. Huang, J.S. Zhu. J. Phys. D 45, 385001 (2012).

[17] H. Sun, Yu. Wu, T. Yao, Yu. Lu, H. Shen, F. Huang, X. Chen. J. Alloys Compd. 765, 27 (2018).

[18] W.H. Press, S.A. Teukolsky. Comp. Phys. 4, 669 (1990).

[19] M. Matsuda, R.S. Fishman, T. Hong, C.H. Lee, T. Ushiyama, Y. Yanagisawa, Y. Tomioka, T. Ito. Phys. Rev. Lett. 109, 067205 (2012).

[20] R. Swanepoel. J. Phys. E Sci. Instrum. 16, 12, 1214 (1983).

[21] V. Sluka, T. Schneider, R.A. Gallardo, A. Kákay, M. Weigand, T. Warnatz, R. Mattheis, A. Roldán-Molina, P. Landeros, V. Tiberkevich, A. Slavin, G. Schütz, A. Erbe, A. Deac, J. Lindner, J. Raabe, J. Fassbender, S. Wintz. Nat. Nanotechnol. 14, 328 (2019).

[22] S. Sergeenkov, N.S. Souza, C. Speglich, V.A.G. Rivera, C.A. Cardoso, H. Pardo, A.W. Mombr, F.M. Araujo-Moreira. J. Phys.: Condens. Matter. 21, 495303 (2009).

Редактор Т.Н. Василевская 